\title{
Efficient Large-Scale Multiplexing of Fiber Bragg Grating and Fiber Fabry-Pérot Sensors for Structural Health Monitoring Applications
}

\author{
G.A.Cranch $^{\mathrm{a}^{*}}$, G.M.H.Flockhart ${ }^{\mathrm{a}}$, C.K.Kirkendall ${ }^{\mathrm{b}}$ \\ ${ }^{a}$ SFA Inc, Crofton, MD 21114, \\ ${ }^{\mathrm{b}}$ Naval Research Laboratory, Code 5674, Washington DC 20375
}

\begin{abstract}
Fiber Bragg gratings have been demonstrated as a versatile sensor for structural health monitoring. We present an efficient and cost effective multiplexing method for fiber Bragg grating and fiber Fabry-Pérot sensors based on a broadband mode-locked fiber laser source and interferometric interrogation. The broadband, pulsed laser source permits time and wavelength division multiplexing to be employed to achieve very high sensor counts. Interferometric interrogation also permits high strain resolutions over large frequency ranges to be achieved. The proposed system has the capability to interrogate several hundred fiber Bragg gratings or fiber Fabry-Pérot sensors on a single fiber, whilst achieving sub-microstrain resolution over bandwidths greater than $100 \mathrm{kHz}$. Strain resolutions of $30 \mathrm{n} \varepsilon / \mathrm{Hz}^{1 / 2}$ and 2 $\mathrm{n} \varepsilon / \mathrm{Hz}^{1 / 2}$ are demonstrated with the fiber Bragg grating and fiber Fabry-Pérot sensor respectively. The fiber Fabry-Pérot sensor provides an increase in the strain resolution over the fiber Bragg grating sensor of greater than a factor of 10 . The fiber Bragg gratings are low reflectivity and could be fabricated during the fiber draw process providing a cost effective method for array fabrication. This system would find applications in several health monitoring applications where large sensor counts are necessary, in particular acoustic emission.
\end{abstract}

Keywords: Strain sensor, multiplexing, acoustic emission, fiber Bragg grating, mode-locked laser

\section{INTRODUCTION}

Multiplexed fiber Bragg grating strain sensor systems have been demonstrated in many different forms since the early 1990s. The potential of fiber Bragg gratings (FBG) as sensing elements in a smart structure was recognized early on [1]. The ability of the FBG sensor array to be embedded into a composite structure with minimal impact on the structural integrity and immunity from electro-magnetic interference, generated considerable interest in the research community. Consequently many multiplexing systems have been developed. Systems generally differ from one another in terms of the method used to interrogate the sensor arrays and leads to variations in performance, cost and number of sensors that can be simultaneously interrogated. In most cases a system can be divided into two parts. One part is the method used to distinguish one sensor from another, such as wavelength division multiplexing (WDM), time division multiplexing (TDM) or code division multiplexing (CDM), for example. The other part relates to how the wavelength of the FBG is measured, sometimes known as the wavelength decoder. For example such methods may use a scanning tunable filter, differentiator and zero-crossing circuit, a CCD spectrometer or fiber-optic interferometer. In most cases, the multiplexing method will determine how many sensors can be simultaneously interrogated and the wavelength decoder will determine the strain sensitivity or resolution and drift of the measurement. For most applications strain resolutions of the order $\pm 1 \mu \varepsilon$ and long term drift below $\sim 10 \mu \varepsilon$ are usually adequate and readily achievable. Measurement bandwidths are usually the order of a few hundred Hertz. However, some specialist applications, such as those described below, require more demanding performance and thus careful attention must be paid to the design of the system. Table 1 summarizes various fiber Bragg grating strain sensor systems reported in the open literature. Although this is not intended as an exhaustive list, the systems described are considered to provide a good combination of sensor resolution and array size.

There are many systems capable of interrogating up to 16-30 sensors on a single fiber but for multiplexing greater than

*Email: geoff.cranch@nrl.navy.mil

Advanced Sensor Technologies for Nondestructive Evaluation and Structural Health Monitoring II, edited by Norbert Meyendorf, George Y. Baaklini, Bernd Michel,

Proc. of SPIE Vol. 6179, 61790P, (2006) -0277-786X/06/\$15 - doi: 10.1117/12.657416

Proc. of SPIE Vol. $617961790 \mathrm{P}-1$ 
50 sensors, systems based on TDM [3,20,26], a combination of WDM and TDM [19] or optical frequency domain reflectometry (OFDR) [16] are required. Although not demonstrated yet, other systems based on dense (D)WDM and CDM [22] or coherence [25] may also be capable of interrogating large arrays. As mentioned previously, the sensor resolution is primarily determined by the wavelength decoding method and although resolutions less than $1 \mu \varepsilon$ are readily achievable, achieving strain resolutions much less than $1 \mu \varepsilon$ requires careful design. Even if high resolution is obtained, low frequency drift can vary considerably. One source of drift is due to the intrinsic temperature sensitivity of the FBG. However, if we consider measuring the differential strain between two closely located FBGs, drift is usually still observed. Measurement drift of this kind will affect both the measurement repeatability and accuracy. Although the causes of drift are varied, the sources are usually related to temperature sensitivity of the components in the wavelength decoder and/or polarization related effects. It is these sources of drift that will be considered in more detail for the proposed interrogation system.

Table 1: Review of high-performance multiplexing methods for Bragg grating strain sensor systems

\begin{tabular}{|c|c|c|c|c|c|c|c|c|}
\hline $\begin{array}{l}\text { Multiplexing } \\
\text { method }\end{array}$ & $\begin{array}{c}\text { Demodulation } \\
\text { method }\end{array}$ & 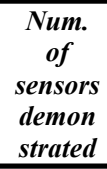 & $\begin{array}{c}\text { Potential } \\
\text { num. of } \\
\text { sensors/ } \\
\text { fiber }\end{array}$ & Strain & esolution & Bandwidth & Source & Ref \\
\hline & & & estimated & $\mathrm{DC}(\mu \varepsilon)^{* *}$ & $\mathrm{AC}(\varepsilon)$ & $\mathrm{Hz}$ & & \\
\hline TDM & Edge-filter & 16 & 16 & & $2 \mathrm{n}(300 \mathrm{~Hz})$ & $>500$ & MLL & 2,3 \\
\hline TDM & Edge-filter & $100^{*}$ & 100 & & $\begin{array}{c}37 \mathrm{n} \\
(300 \mathrm{~Hz})\end{array}$ & $>500$ & SOA & 4 \\
\hline TDM & $\begin{array}{l}\text { Interferometric } \\
\text { (Serrodyne) }\end{array}$ & 8 & 16 & 1.2 & $\begin{array}{c}220 \mathrm{n} \\
(30 \mathrm{~Hz})\end{array}$ & 30 & $\begin{array}{c}\text { SLD } \\
(830 \mathrm{~nm})\end{array}$ & 5,6 \\
\hline $\begin{array}{c}\text { WDM/ } \\
\text { Scanning FP }\end{array}$ & $\begin{array}{l}\text { Interferometric } \\
\qquad(3 * 3)\end{array}$ & 4 & 16 & & $4 \mathrm{n}(0.1 \mathrm{~Hz})$ & 10000 & SLD (1550) & 7,8 \\
\hline WDM & $\begin{array}{l}\text { Interferometric } \\
\text { (Serrodyne) }\end{array}$ & 4 & 16 & & $90 \mathrm{n}(7 \mathrm{~Hz})$ & & EDFA & 8 \\
\hline TDM & & & 20 & 3 & & & $\begin{array}{c}\text { Tunable } \\
\text { laser+IOM }\end{array}$ & 9 \\
\hline AOTF/TDM & Interferometric & 8 & 8 & & $11 n$ & $>10000$ & EDFA & 10 \\
\hline FMCW & $\mathrm{RF} / \mathrm{TOF}$ & 3,6 & $\begin{array}{c}32 \\
(>100)\end{array}$ & $2(\mathrm{rms})$ & & & & 11 \\
\hline TDM & $\begin{array}{l}\text { Mono- } \\
\text { chromator }\end{array}$ & 3 & & 3.4 & & & $\begin{array}{l}\text { Pulsed laser } \\
\text { diode }\end{array}$ & 12 \\
\hline TDM & Interferometric & 4 & & & $2 \mathrm{n}(100 \mathrm{~Hz})$ & & $\begin{array}{l}\text { LED and } \\
\text { ext. mod }\end{array}$ & 13 \\
\hline $\begin{array}{c}\text { WDM/ } \\
\text { Spectrometer }\end{array}$ & Interferometric & 4 & 16 & $0.1 \mathrm{~K}$ & & Low & SLD & 14 \\
\hline $\begin{array}{l}\text { WDM } \\
\text { OFDR }\end{array}$ & Interferometric & $\begin{array}{c}4 \\
800\end{array}$ & $\begin{array}{c}16 \\
800\end{array}$ & 1 & $1.5 \mathrm{n}$ & $\begin{array}{l}2000 \\
\text { Low }\end{array}$ & $\begin{array}{c}\text { EDFA } \\
\text { TL }\end{array}$ & $\begin{array}{l}15 \\
16\end{array}$ \\
\hline WDM & $\mathrm{CCD}$ & 30 & & 1 & $<10 \mathrm{n}$ & $\begin{array}{c}1000 \\
(10000)\end{array}$ & $\begin{array}{c}\text { SLD } \\
(830 \mathrm{~nm})\end{array}$ & 17 \\
\hline WDM & $\mathrm{CCD}$ & 3,2 & $30 \times \mathrm{n}$ & 3 & & $190-10000$ & $\begin{array}{c}\text { SLD } \\
(830 \mathrm{~nm})\end{array}$ & 18 \\
\hline WDM & Scanning FP & 12 & 12 & 1 & $1 \mu$ & $\sim 100$ & ELED (1.3) & 19 \\
\hline WDM/TDM & & 3 & 1000 & & & & $\begin{array}{c}\text { MLL } \\
(1550)\end{array}$ & 20 \\
\hline IWDM & Scanning FP & 10 & 60 & 1 & & 50 & $\begin{array}{l}\text { EDFA (req. } \\
\text { dual peak } \\
\text { FGBs) }\end{array}$ & 21 \\
\hline $\begin{array}{c}\text { DWDM/ } \\
\text { CDMA }\end{array}$ & & 2 & 100 & & & & TL & 22 \\
\hline FDM & & & & 0.19 & & & SLD & 23 \\
\hline $\begin{array}{l}\text { TDM/WDM } \\
\text { Coherence }\end{array}$ & Scanning filter & 16 & $\begin{array}{l}16 \times \mathrm{n} \\
\text { Large }\end{array}$ & 10 & & & $\begin{array}{l}\text { BBS } \\
\text { LD }\end{array}$ & $\begin{array}{l}24 \\
25\end{array}$ \\
\hline $\begin{array}{c}\text { TDM } \\
\text { (Insensys) }\end{array}$ & Edge-filter & 30 & 100 & $1-5$ & $<1 \mu$ & 500 & SOA & 26 \\
\hline
\end{tabular}

${ }^{*}$ Only one sensor read-out at a time ${ }^{* *}$ Measurement times vary - typically few mins

[Key: FP - Fabry-Pérot; FMCW - frequency modulated continuous wave; IWDM - Intensity and WDM; TOF - tunable optical filter; SOA - semiconductor optical amplifier; EDFA - erbium doped fiber amplifier; LED - light emitting diode; ELED - edge emitting LED; TL - tunable laser; BBS - broad-band source; LD - laser diode; MLL -mode-locked laser] 
Three specific applications are considered in this manuscript: (i) the smart composite wing or panel containing an embedded array of strain sensors capable of generating a strain map of the structure, (ii) a shape correcting panel containing an array of shape or curvature sensors and (iii) a health monitoring system based on acoustic emission. These applications require a combination of very high multiplexing gain ( $>50$ sensors/fiber), sub-microstrain resolution, very low drift, low crosstalk and/or large bandwidth $(>100 \mathrm{kHz})$. The present work describes a configuration capable of achieving these requirements by combining a broadband, mode-locked erbium doped fiber laser source (MLL) with interferometric interrogation. The MLL configuration can generate broadband (greater than $60 \mathrm{~nm}$ ), square optical pulses of duration 10 to 30 nanoseconds (tunable by pump power and fiber ring birefringence), peak pulse powers approaching $1 \mathrm{~W}$, at a repetition rate of several hundred kiloHertz [27]. The spectral emission properties of this laser have been investigated further in [28], which demonstrates this laser to be well suited to high performance interrogation of FBG sensors. The peak power produced by this MLL exceeds that of a super-luminescent diode (SLD) and semiconductor optical amplifier (SOA) by greater than two orders of magnitude. It is powered by a single pump diode and is therefore more efficient than the externally modulated erbium doped fiber amplifier (EDFA) source.

In the proposed configuration, a serially multiplexed array of FBG sensors consists of low reflectivity FBGs each at a different wavelength. This sequence of FBGs is repeated periodically along a single fiber. A low cost method of fabricating multiplexed FBG arrays is based on single pulse grating fabrication on the draw tower [29]. Continued development of this technique has considerably improved the quality of the FBGs over the initial demonstrations and may make possible on-line fabrication of apodized FBGs at pre-determined wavelengths. Alternatively, other techniques based on strip and recoat [30] or by writing through the fiber coating [31] are currently available. The proposed configuration is also demonstrated to interrogate fiber Fabry-Pérot cavities (FFP), which provide greater than an order of magnitude increase in strain sensitivity. These can be fabricated in the same way as multiplexed arrays of FBG sensors.

This paper is arranged as follows. The mode-locked laser and multiplexing scheme are described in section 2 . The FBG and FFP sensor system performance are presented in section 3. Section 4 discusses applications of this system. Finally, a summary is given in section 5 .

\section{MULTIPLEXING SCHEME}

\subsection{Mode-locked laser}

The MLL comprises a unidirectional ring cavity illustrated in fig. 1(a). The principle of the passive mode-locking scheme relies on nonlinear polarization switching, where the beat length of the optical fiber within the ring is power dependent, giving rise to a power dependence of the transmission. This yields a stable operating regime consisting of the emission of square-shaped optical pulses. Operation in this so-called multi-beatlength regime results in mode-locked behavior occurring at relatively low pump powers [32]. Stimulated Raman scattering broadens the emitted radiation beyond the erbium window to greater than $60 \mathrm{~nm}$. The MLL characterized here was first reported in [27]. The ring contains a length of erbium doped fiber, pumped by a $980 \mathrm{~nm}$ laser diode, approximately $700 \mathrm{~m}$ of dispersion-shifted fiber, a polarizing isolator (P-ISO) and two polarization controllers (PC). The polarization controllers provide the birefringence control required to obtain the mode-locked regime.

Once the mode-locked regime is obtained, the pulse width is typically on the order of $10 \mathrm{~ns}$ and the repetition frequency, $f_{\text {rep }}$, is $278 \mathrm{kHz}$ (or period of $3.48 \mu \mathrm{s}$ ) corresponding to one cavity round trip time (cavity fiber length 736 $\mathrm{m})$. The optical duty cycle (DC) is therefore $2.8 \times 10^{-3}(-25.5 \mathrm{~dB})$. The peak emission power density of the laser is shown in fig. 1. Thus, the launched pulse power density is $7 \mathrm{dBm}$ at $1550 \mathrm{~nm}$ in a $0.2 \mathrm{~nm}$ bandwidth. The optical extinction ratio (defined as the ratio of the 'on' to 'off' power levels) of the MLL is greater than $2222(33.5 \mathrm{~dB})$. The relative intensity noise (RIN) measured in a $0.23 \mathrm{~nm}$ optical bandwidth over the wavelength range $1525 \mathrm{~nm}$ to $1575 \mathrm{~nm}$ is -79 $\mathrm{dB} / \mathrm{Hz} \pm 4 \mathrm{~dB}$ at frequencies above $10 \mathrm{kHz}$ and increases to $-63 \mathrm{~dB} / \mathrm{Hz} \pm 7 \mathrm{~dB}$ at frequencies less than $100 \mathrm{~Hz}$. The error bounds specified here indicate the measured variation with wavelength.

The output emission is polarized with a degree of polarization of $\sim 74 \%$ measured with a Stokes analyzer. To depolarize the emission of the MLL, a Lyot depolarizer (LDPOL or LD) is used. The LDPOL is designed to depolarize the light 
from the spectrally narrowed reflection of the FBG sensor with a full-width half-maximum of $0.2 \mathrm{~nm}$. The LDPOL is constructed from two sections of PM PANDA ${ }^{\text {TM }}$ fiber (beat-length $3.9 \mathrm{~mm}$ at $1550 \mathrm{~nm}$ ) of length $102 \mathrm{~m}$ and $500 \mathrm{~m}$ with their fast axes spliced at $45^{\circ}$ (note that the second section need only be $204 \mathrm{~m}$ in length for correct operation). Placing the LDPOL at the output of the MLL reduces the degree of polarization to less than $5 \%$.

(a)

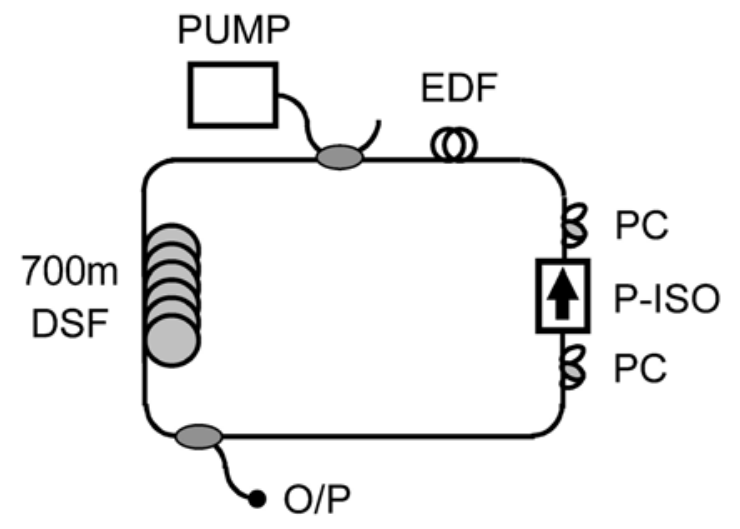

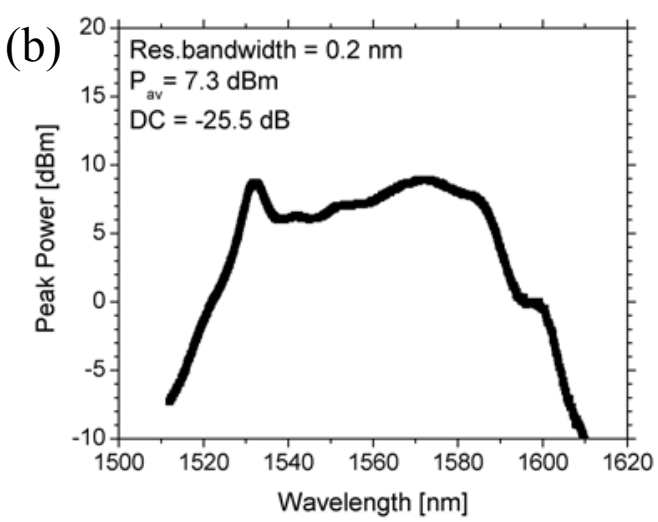

Figure 1(a) Mode-locked laser configuration and (b) output spectrum

\subsection{Interferometric interrogation scheme}

\subsubsection{Drift compensated Michelson interferometer interrogation of Bragg gratings}

The sensor system configuration is based on a concept first proposed in [35] and is illustrated in fig. 2.

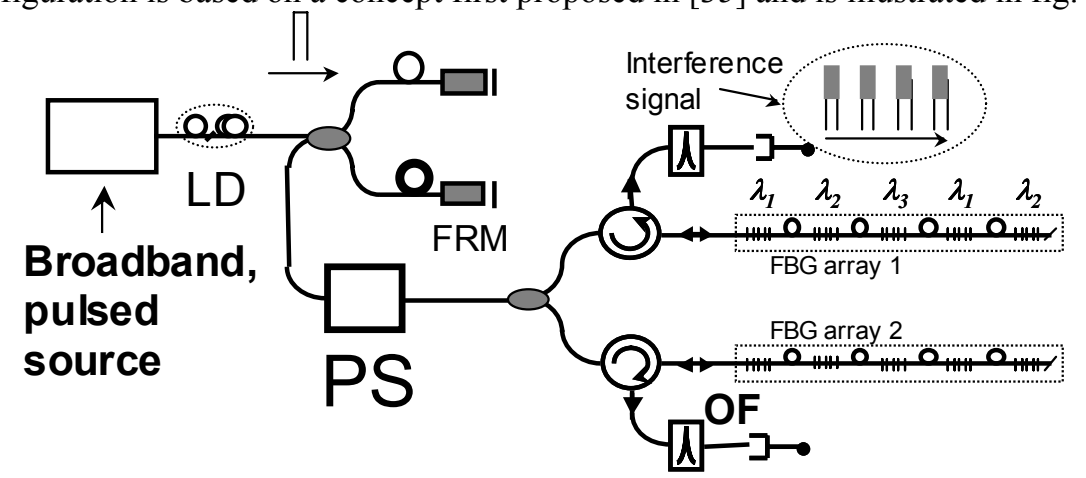

Figure 2. Sensor system configuration

The output from the MLL is launched into a fiber-optic Michelson interferometer (MI) with a $\sim 5 \mathrm{~mm}$ fiber path imbalance, which is comparable to the coherence length of the light reflected from a sensor grating. Each arm of the MI is terminated with a Faraday reflector mirror (FRM) to reduce effects of birefringence in the fiber. The output from the MI is split into two and injected into the FBG sensor array. Each FBG in the array produces a reflection of the incident radiation, which is a spectral slice over the grating reflection band. The reflected signals are separated with a circulator (CIRC) or directional coupler. Two FBG arrays are thus interrogated simultaneously. The fiber length between each FBG in the experimental system is $91 \mathrm{~m}$. The FBGs are written in SMF- $28^{\mathrm{TM}}$ fiber and are $\sim 5 \mathrm{~mm}$ in length apodized by the Gaussian laser beam profile. The FBGs reflectivities are typically on the order of $1 \%$ and have full-width halfmaximums of $0.2 \mathrm{~nm}$. FBGs are numbered 1 to 4 in FBG array 1 and 5 to 8 in FBG array 2. The center wavelength of FBG 1,2,5 and 6 are 1549.6nm and FBG 3,4,7 and 8 are $1546.6 \mathrm{~nm}$. The signals returning from each array thus consist of a temporal pulse train, where each pulse corresponds to an FBG sensor and consists of a fringe pattern, the phase of which contains the strain information of interest. The MLL tap (not shown) is used to generate a trigger pulse for the pulse generator (PG), which generates a pulse train with a controllable delay to trigger the analog to digital converter (A/D). Thus a single sample is acquired during each pulse return from the FBG array and the signals from all four FBG sensors are recorded by a single A/D. Once digitized, the fringe pattern can be processed to obtain the strain 
information. The individual FBG sensor signals are extracted during the signal processing. High speed (5 MHz) PINFET receivers are used for detecting the return from each FBG array.

The phase generated carrier (PGC) interrogation technique is used to extract the interferometric phase and hence the Bragg wavelength of the FBG sensor [33]. In this technique, a sinusoidal phase modulation is applied to one of the arms of the MI. The processing stages for this method are now briefly described. It is assumed that the power split ratio of the directional coupler in the $\mathrm{MI}$ is $50 \%$ and there is no excess loss in either interferometer arm. The current generated by the photodetector during a return pulse is then given by,

$$
i_{p h}=R P\left(1+V \cos \left[\phi_{p g c} \cos \omega_{p g c} t+\Delta \phi(t)\right]\right)
$$

where $R$ is the photodiode responsivity, $P$ is the peak return power in the absence of the interference term, $V$ is the normalized fringe visibility, $\phi_{p g c}$ is the modulation depth, and $\Delta \phi(t)$ includes signal and drift phases. Extraction of the phase signal is shown diagrammatically in fig. 3 .

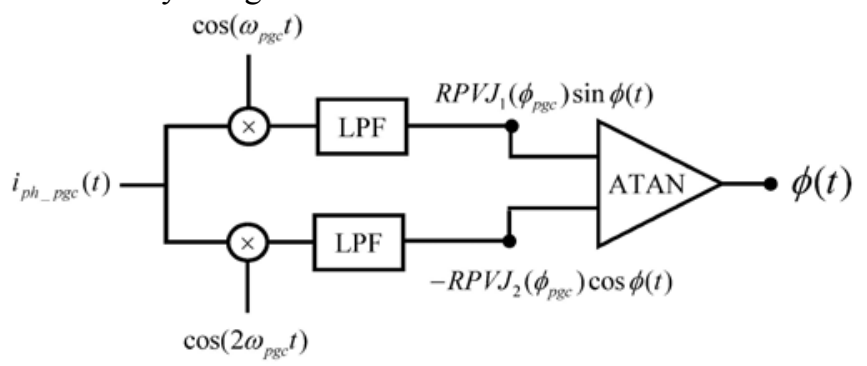

Figure 3. Processing steps required for extraction of the phase

Thus, quadrature components of the phase of interest, $\Delta \phi(t)$, can be obtained by synchronous detection of the photodiode current at $\omega_{p g c}$ and $2 \omega_{p g c}$ and low pass filtering the resulting signals. The phase is obtained by normalizing the amplitudes of the filtered signals and taking the arctangent of their ratio. Setting $\phi_{p g c}$ equal to 2.6 radians results in $J_{1} \approx J_{2}$; however, a suitable normalization routine is also applied in the signal processing to remove any small differences in amplitude. Phase excursions greater than pi radians can be measured by implementing a fringe counting algorithm. The accuracy to which the amplitudes of the filtered signals are matched determines the phase measurement accuracy. A PGC frequency of $3 \mathrm{kHz}$ has been used for the results presented here. This modulation is imparted onto the optical signal in one arm of the MZI with a fiber wrapped piezoelectric tube phase modulator (PZT).

A change in wavelength of the FBG causes a change in interferometric phase given by, $\Delta \phi_{F B G}=4 \pi n d_{M I} \Delta \lambda / \lambda_{F B G}^{2}$ where $n$ is the fiber effective refractive index, $d_{M I}$ is the interferometer fiber path imbalance and $\lambda_{F B G}$ is the Bragg wavelength of the FBG. The fractional change in wavelength with applied strain is given by $\Delta \lambda / \lambda_{F B G}=0.78 \Delta \varepsilon$, where the factor of 0.78 includes the contribution to the Bragg wavelength shift from the stress-optic effect [34]. The phase is thus linearly related to the MI path imbalance and strain applied to the FBG, $\Delta \varepsilon$, by,

$$
\Delta \phi_{F B G}=\frac{4 \pi n d_{M I}}{\lambda_{F B G}} 0.78 \Delta \varepsilon
$$

The bandwidth of the optical emission from the MLL is approximately 60nm, thus, FBG sensors can also be multiplexed with wavelength division multiplexing. An optical filter (OF) placed before the photodetector is used to separate the signals from FBGs at different wavelengths. The use of TDM with WDM greatly increases the number of sensors that can be multiplexed onto a signal fiber.

\subsubsection{Drift compensated Michelson interferometer interrogation of fiber Fabry-Pérot cavities}

A second configuration is based on interrogating fiber Fabry-Pérot sensors. The FFP consists of two low reflectivity $(r \ll 1)$ FBGs formed a few centimeters apart, thus forming a low finesse Fabry-Pérot cavity. The path imbalance in the 
MI is matched to the FFP forming a tandem interferometer arrangement. The FBG array in fig. 2 is thus replaced by a series of FFP sensors. The current generated by the photodetector during a return pulse (for $r \ll 1$ ) is given by,

$$
i_{p h} \simeq R P\left(1+\frac{V}{2} \cos \left[\phi_{p g c} \cos \omega_{p g c} t+\Delta \phi_{F F P}(t)\right]\right)
$$

where $\phi_{F F P}(t)$ is the phase of the FFP cavity (note that this phase will also include phase shifts generated in the MI). The amplitude of the interference signal is thus reduced by 0.5 compared with the FBG sensor response. For the case when $d_{F F P}=d_{M I}$, the strain applied, $\Delta \varepsilon$, to the FFP sensor is related to the measured phase shift by,

$$
\Delta \phi_{F F P}=\frac{4 \pi n d_{F F P}}{\lambda_{F B G}} 0.78 \Delta \varepsilon
$$

For the case when $n=1.468$ and $d_{M I}=3.3 \mathrm{~mm}$ for the FBG sensor and $d=6 \mathrm{~cm}$ for the FFP sensor then taking the ratio of (4) with (2) yields $\Delta \phi_{F F P} / \Delta \phi_{F B G}=d_{F F P} / d_{F B G}=18$. Thus the FFP sensing exhibits a responsivity $\sim 18$ times higher than the FBG sensor.

These configurations are ideally suited to differential strain measurements between two FBG or FFP sensors, as demonstrated in [35]. Phase shifts generated in the MI will be common mode to both FBGs or FFPs and can therefore be rejected.

\subsection{3. $3 \times 3$ coupler interrogation}

For high frequency applications $(>\sim 20 \mathrm{kHz})$ a different interferometric arrangement must be used. A suitable configuration is shown in fig. 4 .

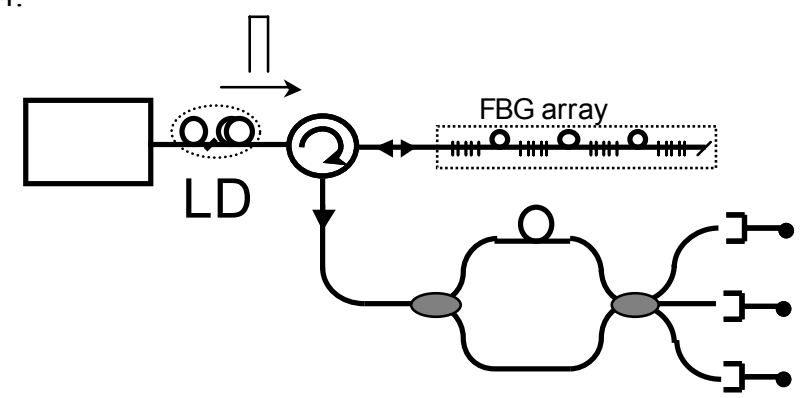

Figure 4: Sensor system configuration

In this case the light from the MLL is launched directly into the FBG (or FFP) array. The return signals from the array are launched into a Mach-Zehnder interferometer (MZI) with a $3 \times 3$ coupler. The three outputs from this coupler provide a means of extracting the phase of the interferometer without the need for applying a phase modulation carrier. The outputs of the interferometer are of the form:

$$
\begin{aligned}
& i_{p h_{-} 1}=R P\left(1+V \cos \left[\Delta \phi(t)+\phi_{1}\right]\right) \\
& i_{p h_{-} 2}=R P\left(1+V \cos \left[\Delta \phi(t)+\phi_{2}\right]\right) \\
& i_{p h_{-} 3}=R P\left(1+V \cos \left[\Delta \phi(t)+\phi_{3}\right]\right)
\end{aligned}
$$

Characterizing the power split ratio of the coupler yields the phase coefficients of the coupler, $\phi_{1}-\phi_{3}$. The phase signal of interest can then be extracted using the method described in [36]. Since the output of the $3 \times 3$ contains the phase information at baseband (i.e. no down-conversion is required in the processing) then the signal bandwidth is equal to half the sample rate of the three detector. 


\section{SYSTEM PERFORMANCE}

\subsection{Based Wavelength Decoder}

\subsubsection{Strain resolution}

The strain resolution achieved with the multiplexed array of 4 FBG sensors, described in sec. 2.1.1, is shown in fig. 5(a). The strain resolution is between 23 to $60 \mathrm{n} \varepsilon / \mathrm{Hz}^{1 / 2}$ at frequencies below $10 \mathrm{~Hz}$. This corresponds to a phase resolution ranging from 0.38 to $1 \mathrm{mrad} / \mathrm{Hz}^{1 / 2}$. This is limited by relative intensity noise of the MLL [37] and is about an order of magnitude higher than the strain resolution achieved with a standard erbium doped fiber source [35]. When two arrays of FBGs are present, the differential strain between two corresponding FBGs can be measured, as shown in fig. 5(b). The stability of this differential strain gives a measure of the differential strain accuracy. Polarization related effects often cause a small degree of drift when the connecting leads are perturbed [35]; however, by use of a Lyot depolarizer in the output of the MLL, this drift has been reduced to $\pm 1 \mu \varepsilon$. The arrow in fig. $5(\mathrm{~b})$ indicates the time at which the connecting leads are perturbed. The resulting strain drift is observed in the differential strain measurement.
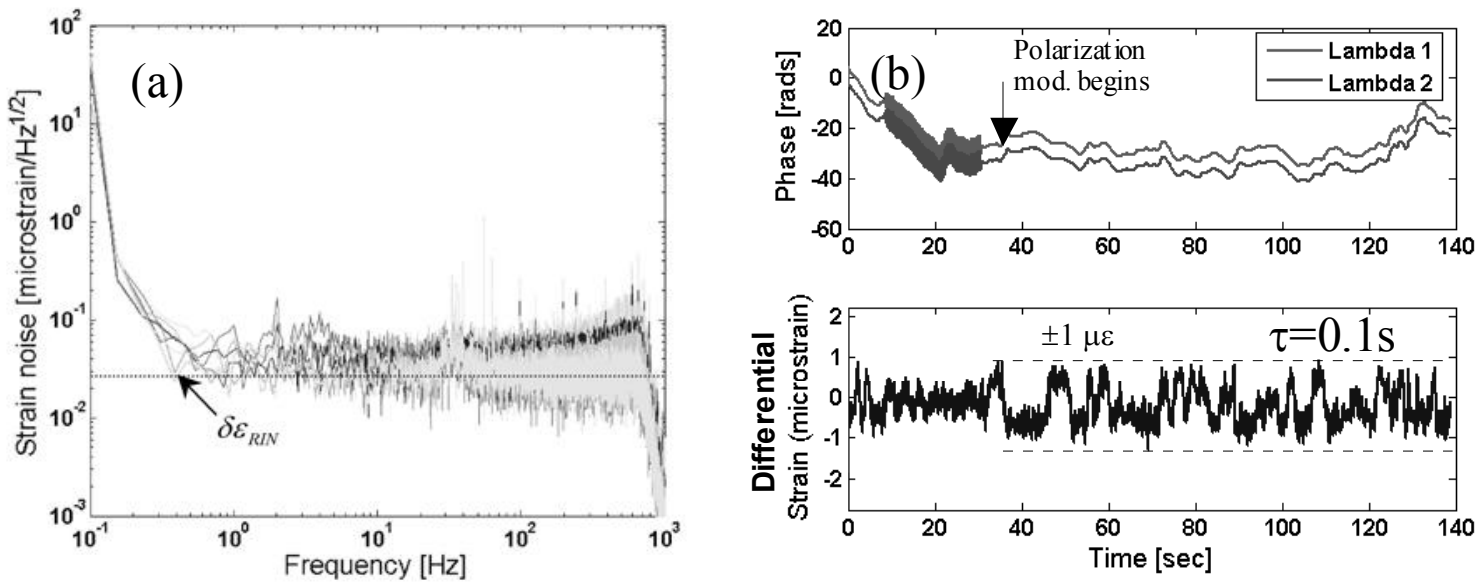

Figure 5(a) FBG 1-8 strain resolution (b) (top) Phase corresponding to each FBG and (bottom) differential strain drift between two co-located FBGs (note that the signal between $8 \mathrm{sec}$ and $30 \mathrm{sec}$ is a calibration tone)
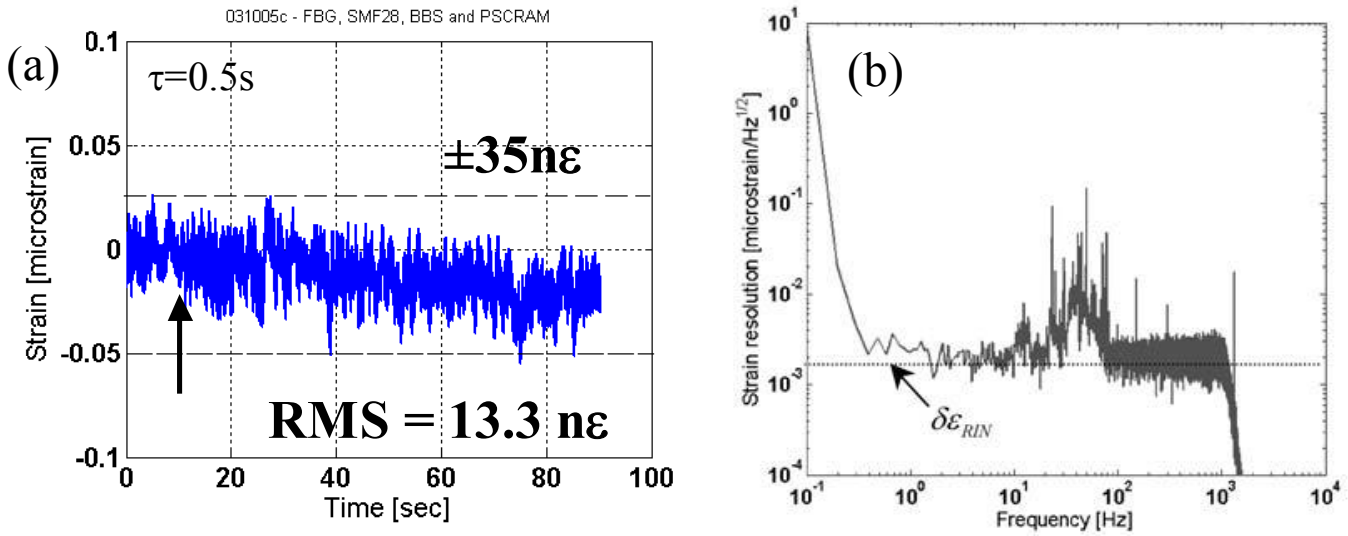

Figure 6(a) Drift when polarization scrambling is used and (b) Strain resolution of single FFP sensor interrogated with MLL

This level of drift can be further reduced by adding a polarization scrambler (PS) to the output of the MI in fig. 2. An experiment conducted on a single FBG with an erbium doped fiber source demonstrates that differential strain drift due to polarization related effects can be reduced to less than $\pm 35 \mathrm{n} \varepsilon( \pm 0.6 \mathrm{mrad})$, as shown on fig. 6(a). The arror in fig. 6(a) 
indicates when the lead perturbation begins. This experiment yielded an rms strain noise of $13.3 \mathrm{n} \varepsilon$ measured with an integration time of $0.5 \mathrm{sec}$. For a detailed investigation of polarization related drift effects see ref. [38].

The strain resolution for a single FFP sensor interrogated with the MLL system is shown in fig. 6(b). The strain resolution achieved is $2 \mathrm{n} \varepsilon / \mathrm{Hz}^{1 / 2}\left(1.1 \mathrm{mrad} / \mathrm{Hz}^{1 / 2}\right)$ also limited by relative intensity noise from the MLL. Thus the FFP sensor provides an increase in the strain resolution of approximately an order of magnitude.

\subsubsection{Crosstalk}

Crosstalk arises from three sources: (a) multipath reflections, (b) leakage light and (c) shadowing. Multipath reflection induced crosstalk occurs from the multiple reflections that occur between FBGs of the same wavelength. Leakage light arises from a finite extinction ratio of the optical source (i.e. the light emitted from the source when in its 'off' state). This will cause light from other sensors to arrive at the detector at the same time as the main pulse. Crosstalk due to shadowing can be considered for the case of an ideal array where the FBG reflectivity profiles are ideal Gaussian lineshapes centered at identical wavelengths. A shift in the wavelength of several sensors by the same amount will cause the power transmitted to the subsequent sensors to change (each FBG sees an incident optical spectrum that is a convolution of the source emission spectrum and the previous FBGs spectra). This may give rise to an apparent change in the measured centre wavelength or phase of the 'shadowed' sensors. This problem is alleviated in a real system if the center wavelengths of the FBGs are not identical and random variations in the reflectivity spectra are present from one FBG to the next. The crosstalk due to the first two sources as a function of the number of sensors and the FBG power reflectivity is shown in fig. 7(a). Crosstalk levels less than $-30 \mathrm{~dB}$ limit the number of time division multiplexed sensors to $\sim 50$.
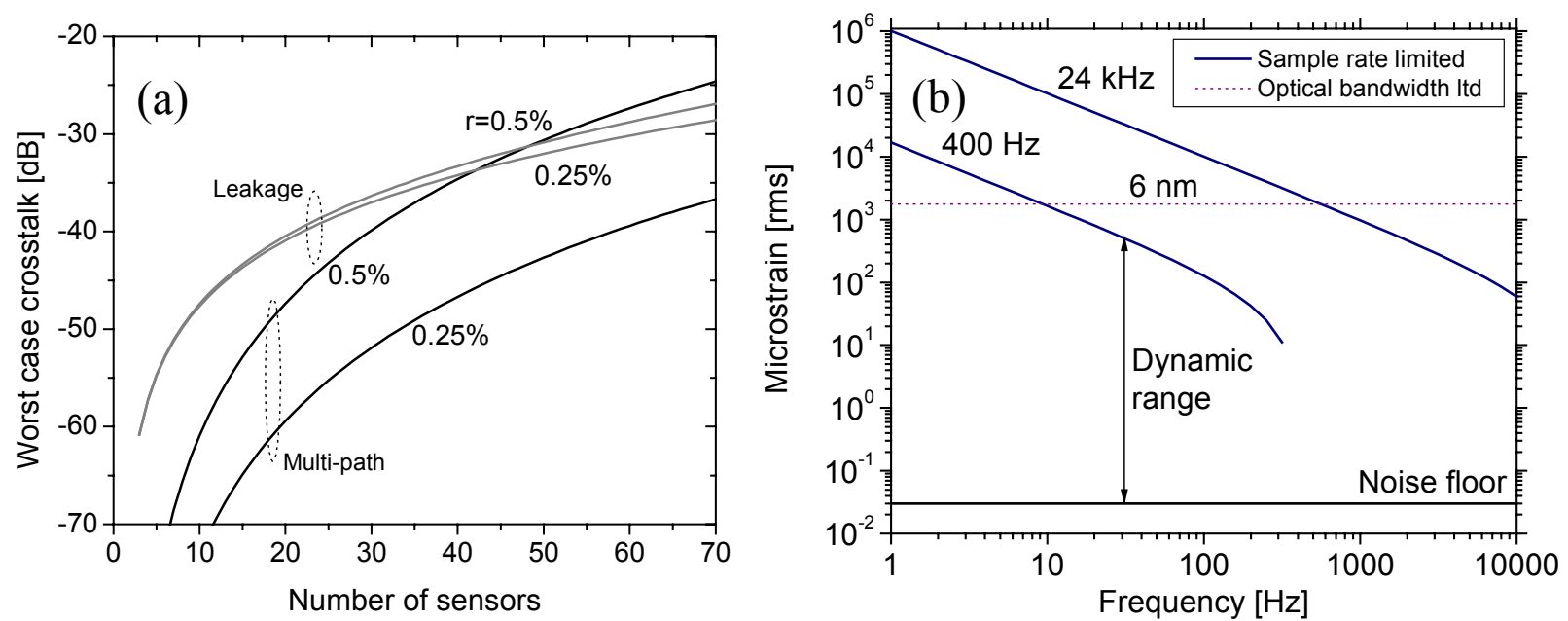

Figure 7(a) Crosstalk due to leakage light and multi-path (worst-case) and (b) Dynamic range of MI configuration

\subsubsection{Sensor bandwidth}

The sensor bandwidth is determined by the maximum frequency signal that can be faithfully reproduced by the signalprocessing algorithm. It is related to the PGC frequency or more specifically, the frequency at which the sine and cosine signals in fig. 3 are low pass filtered. This is usually set to slightly less than half the PGC frequency, in this case $800 \mathrm{~Hz}$ for a PGC frequency of $3 \mathrm{kHz}$. Assuming that the PGC signal has to be over sampled by a factor of 10, then the maximum PGC frequency is $27.8 \mathrm{kHz}$, if the sampling rate is $278 \mathrm{kHz}$, thus the sensor bandwidth can be increased in the present system by an order of magnitude.

For quasi-DC measurements the dynamic range of a sensor is dependent on the optical bandwidth allocation for the sensor when WDM is used. For AC measurements, the dynamic range is also limited by the bandwidth of the signalprocessing algorithm. The dependence of the dynamic range on frequency for sine/cosine filter bandwidths of $400 \mathrm{~Hz}$ and $24 \mathrm{kHz}$ is shown in fig. 7(b). Thus, for low frequency signals $<10 \mathrm{~Hz}$, the dynamic range is $\sim 100 \mathrm{~dB}$, assuming the sensor is optically limited to a bandwidth of $6 \mathrm{~nm}$. 


\subsubsection{Optical power limitations}

It has, thus far, been assumed that the reflectivity of the FBGs is sufficiently low to minimize crosstalk and to maintain a constant return power from each sensor. However, for large number of sensors there will always be some variation in returned power from the sensors due to the depletion of the input optical signal on propagation through the array as well as manufacturing variations in the FBG reflectivities. If the power reflectivity of the FBG is $r$ and we neglect fiber attenuation, then the difference in power between the first sensor and sensor $N$ is $(1-r)^{2(n-1)}$. Thus, for $N=50$ and $r=1 \%$, the maximum power variation is $4.3 \mathrm{~dB}$. This reduces to $1.1 \mathrm{~dB}$ for $r=0.25 \%$. The noise spectral density of the detector without any input power was found to be $\sim 10 \mathrm{~dB}$ below the noise with the optical power connected. Thus, an optical power variation of $4-5 \mathrm{~dB}$ can easily be tolerated in this system without degradation of the sensor performance.

\section{2. $3 \times 3$ based Wavelength Decoder}

The $3 \times 3$ wavelength decoder permits the sample rate limited sensor bandwidth to be achieved. The repetition rate of the MLL determines the maximum sampling rate of the detector signals and is $278 \mathrm{kHz}$. This yields a sensor bandwidth of $139 \mathrm{kHz}$. The power spectral density noise of a single FFP sensor interrogated with a $3 \times 3$ interferometer and an erbium doped fiber source is shown in fig. 8. The outputs of the interferometer are sampled at $300 \mathrm{kHz}$ and yield a noise floor between $112-178 \mu \mathrm{rad} / \mathrm{Hz}^{1 / 2}$ corresponding to a strain resolution of $0.2-0.3 \mathrm{n} \varepsilon / \mathrm{Hz}^{1 / 2}$. A strain resolution approximately an order of magnitude higher would be expected when interrogating with the MLL source. (Note that the spurious tones at $30-40 \mathrm{kHz}$ and $100 \mathrm{kHz}$ are artifacts of our $\mathrm{A} / \mathrm{D}$ converter.)

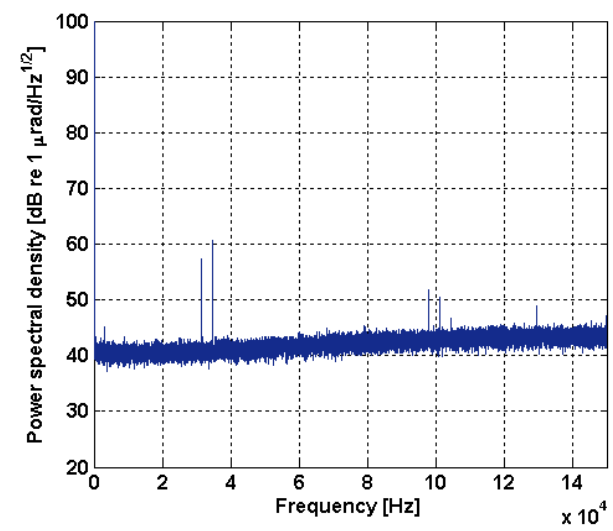

Figure 8 High-frequency phase resolution of FFP sensor measured with $3 \times 3$ coupler MZI

\section{APPLICATIONS}

Discussed below in more detail are three applications for the FBG sensor systems described above.

\subsection{Structural strain mapping}

One of the earliest perceived applications of multiplexed FBG sensors was their ability to provide an internal strain map of the structure in which they are imbedded. This application would not require demanding sensor performance but may require a large number of sensors spaced within a few centimeters of each other [39]. The sensor would be required to measure changes in strain over the operating period of the structure. Strain resolutions of around $1 \mu \varepsilon$ would be adequate over a bandwidth of a few $100 \mathrm{~Hz}$. The proposed configuration provides the capability of small sensor spacing if wavelength and time division multiplexing are both employed. For example, assuming a sample rate of $68.1 \mathrm{MHz}$ time division multiplexing limits the sensor spacing to approximately $3 \mathrm{~m}$; however, if $60 \mathrm{~nm}$ of optical bandwidth is available and each wavelength is allocated $3 \mathrm{~nm}$ of optical bandwidth then 20 wavelengths can be used for WDM, thus reducing the sensor spacing to $\sim 15 \mathrm{~cm}$. 
In the proposed configuration, no absolute wavelength reference is available, thus the strain change can only be measured relative to the strain state at the time of switch-on of the system. For many applications, this may be adequate since it is often of interest to know changes in strain state in the material during operation of the vehicle to which the structure is attached. Reducing the interrogation system size and cost are likely to be very important factors for the success of this application.

\subsection{Shape measurement}

A particularly promising application of the drift compensated MI configuration is a shape sensing array based on multicore fiber curvature sensors [40]. The concept is illustrated in fig. 9(a). FBGs formed in the cores of a multicore fiber experience opposing changes in Bragg wavelength when the fiber is bent (see bottom plot, fig. 9(a)). Measurement of this differential strain yields the curvature of the fiber. The drift compensated MI configuration is a particularly suitable method of interrogating the cores of the MCF-FBG sensor. Phase shifts due to changes in temperature of the MI or the FBGs are cancelled such that the interrogation method provides a drift free measurement of the curvature. This type of sensor is well suited to the real time shape measurement of composite structures. These structures may also include shape memory alloys capable of changing the shape of the structure or to maintain a constant shape. In these applications the measurement rates are likely to be slow, on the order of a few Hertz. However, high phase measurement accuracy is required to achieve high curvature accuracy. Measurements conducted in [40] demonstrate an rms strain measurement accuracy of $\sim 50 \mathrm{n} \varepsilon$ for a $0.5 \mathrm{sec}$ integration time measured over a few minutes. Thus, for a MCF with a core separation of $d=50 \mu \mathrm{m}$, a curvature $(=1 / R)$ measurement accuracy of $1 \mathrm{~km}^{-1}$ is obtained. Some degradation in this accuracy should be expected for a multiplexed system; however, this system would nevertheless provide an extremely high level of shape measurement accuracy.

The response of a single MCF bend sensor to controlled bending is shown in fig. 9(b). For characterization purposes, the MCF sensor is attached to a cantilever, clamped at one end. The other end is displaced with a displacement actuator. The cores of the MCF to be interrogated are aligned with the plane of the applied bending. This causes a well defined amount of curvature to be imposed on the MCF sensor. The response of the MCF sensors to a step-wise increase and decrease in displacement of $1 \mathrm{~mm}$ indicates very good linearity and repeatability.

(a)
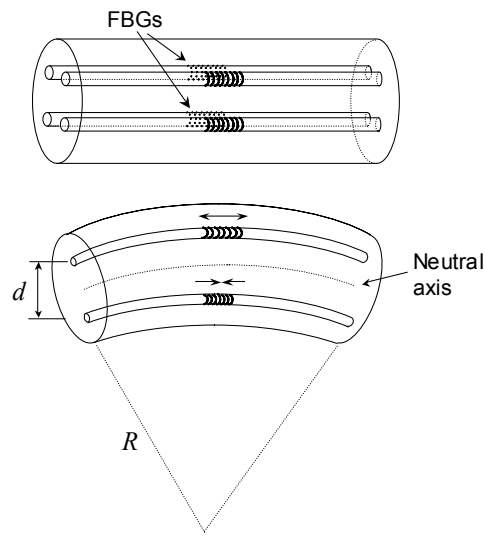

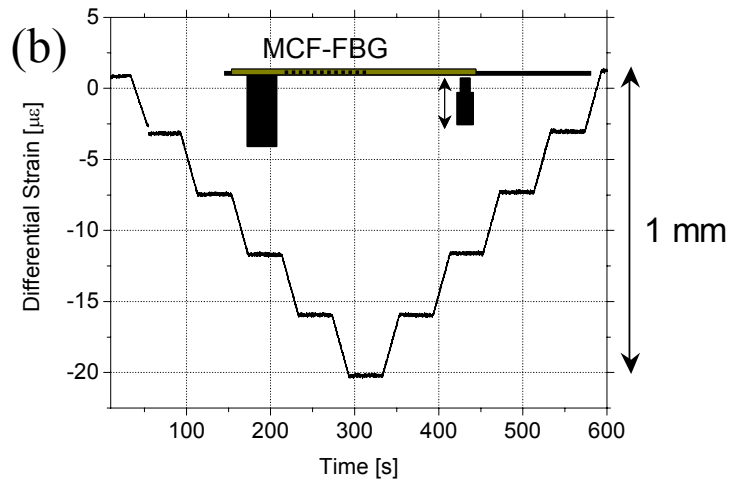

Figure 9: (a) Multicore fiber curvature sensor concept and (b) Measured differential strain vs. displacement of cantilever (inset: MCF on cantilever arrangement)

\subsection{Acoustic emission (AE)}

An attractive application of the FBG sensor is as a means to detect or anticipate mechanical failure of a structure. Energy released during the process of fracture in the material can be detected as a high frequency acoustic signal propagating in the material [41]. Fracture events may simply be counted to monitor structure health [42]; however, spectral analysis could be performed to yield more information. Existing sensors are often based on piezo-electric material that may be tuned to frequencies of a few $\mathrm{MHz}$ where a significant proportion of the acoustic energy exists. However, useful information is available above $100 \mathrm{kHz}$, and may be adequate for an event counting system. The $3 \times 3$ 
interferometer provides a sensor bandwidth of $149 \mathrm{kHz}$ limited by the repetition rate of the MLL; however, this could be increased by reducing the cavity length in the MLL thus increasing the MLL repetition rate. The FFP sensor may be advantageous in providing a higher strain resolution; however, this is at the expense of a longer gauge length device. The orientation of the FBG sensors relative to the direction of propagation of the acoustic wave will also affect the sensitivity. Use of the full bandwidth available would enable the same FBG sensor to measure low-frequency changes in strain for load monitoring as well as high frequency signals due to AE. Some convincing work published to date on the use of FBG sensors for damage detection involves using an excitation method to detect changes in propagating Lamb waves [43] or by the detection of impacts [41]. The detection of internal fractures in a loaded carbon composite cylinder at the time of generation has also been reported using an extrinsic Fabry-Pérot sensor [44].

Reduction of the size of the interrogation system and the overall cost are paramount for this application. Further work is necessary to determine the required sensor performance and configuration; however, the proposed configuration provides considerable flexibility for meeting these requirements.

\section{CONCLUSIONS}

In conclusion we have presented several configurations of a time and wavelength division multiplexed fiber Bragg grating sensor system and reviewed the performance of each configuration. The techniques presented provide a versatile method of interrogating large numbers of multiplexed FBG sensors. Very high performance can be achieved in terms of sensor resolution, dynamic range and measurement bandwidth. Both FBG and FFP sensors can be interrogated with the proposed systems. The FFP sensor provides an increase in the strain resolution of approximately an order of magnitude over the FBG sensors. Three applications are studied in detail: structural strain mapping, multicore fiber shape measurement and acoustic emission and the relative merits of the proposed multiplexing schemes are discussed.

\section{REFERENCES}

[1] R.M.Measures, "Advances toward fiber optic based smart structures", Opt. Eng. 31 (1), pp. 34-47, 1992

[2] D.J.F.Cooper et al, "Time division multiplexing of large serial fiber-optic Bragg grating sensor arrays" Applied Optics, 40(16) 2643-2653, 2001

[3] D.J.F.Cooper, P.W.E.Smith, "Simple high-performance method for large-scale time division multiplexing of fibre Bragg grating sensors", Meas. Sci.Tech, 14, pp.965-974, 2003

[4] Y.J.Rao, A.B.Lobo-Ribeiro, D.A.Jackson, "Combined spatial and time-division-multiplexing scheme for fiber grating sensors with driftcompensated phase-sensitive detection", Optics Lett. 20 (20), pp. 2149-2151, 1995 and Y.J.Rao, "Simultaneous spatial, time and wavelength division mutliplexed in-fibre grating sensing network", Optics. Comm. 125, pp. 53-58, 1996

[5] A.B.Lobo Riberio, Y.J.Rao, L.Zhang, I.Bennion, D.A.Jackson, "Time and spatial multiplexing tree topology for fiber optic Bragg grating sensors with interferometric wavelength shift detection", Applied Optics, 35 (13) pp. 2267-2273, 1996

[6] M.D.Todd, G.A.Johnson, B.L.Althouse, "A novel Bragg grating sensor interrogation system utilizing a scanning filter, a Mach-Zehnder interferometer and a $3 * 3$ coupler" Meas. Sci. Tech., 12, pp. 771-777, 2001

[7] G.A.Johnson, M.D.Todd, B.L.Althouse, C.C.Chang, "Fiber Bragg grating interrogation and multiplexing with a $3 * 3$ coupler and a scanning filter", J.Light.Tech. 18 (8), 2000, pp. 1101-1105

[8] K.Kalli, G.P.Brady, D.J.Webb, D.A.Jackson, L.Zhang, I.Bennion "Wavelength-division and spatial multiplexing using tandem interferometers for Bragg grating sensor networks", Optics Lett. 20 (24), pp. 2544-2546, 1995

[9] C.C.Chan, W.Jin, D.N.Wang, M.S.Demokan, "Performance analysis of a time-division-multiplexed fiber Bragg grating array by use of a tunable laser source", IEEE Sel. Top. Quant. Elec. 6 (5), pp. 741-749, 2000

[10] S.P.Christmas, D.A.Jackson, "A new method for interrogation of serial arrays of dynamic FBG strain sensors", Meas. Sci. Tech., 12, pp. 897900, 2001

[11] P.K.C.Chan, W.Jin, M.Süleyman Demokan, "FMCW Multiplexing of fiber Bragg grating sensors", IEEE J.Sel.Topics in Quant. Elec. 6(5), pp. 756-763, 2000

[12] A.Wilson, S.W.James, R.P.Tatam, "Time-division-multiplexed interrogation of fibre Bragg grating sensors using a laser diode" Meas. Sci.Tech. 12, pp. 181-187, 2001

[13] R.S.Weis, A.D.Kersey, T.A.Berkoff, "A four-element fiber grating sensor array with phase-sensitive detection", IEEE Phot. Tech.Lett. 6 912) pp.1469-1471, 1994

[14] Y.J.Rao, D.J.Webb, "High-resolution, wavelength-division-multiplexed in-fibre Bragg grating sensor system" Elec. Lett. 32 (10) pp. 924-925, 1996

[15] T.A.Berkoff, A.D.Kersey, "Fiber Bragg grating array sensor system using a bandpass wavelength division multiplexer and interferometric detection" IEEE Phot. Tech. Lett. 8 (11), pp. 1522-1524, 1996

[16] M.Froggatt, Moore, "Distributed measurement of static strain in an optical fiber using multiple Bragg gratings at nominally equal wavelengths" Applied Optics, 37 (10), 1998 and M.Froggatt, B.Childers, J.Moore, T.Erdogan, "High density strain sensing using optical frequency domain reflectometry", OFS 2000, SPIE 4185, pp. 249 WE 4-1, 2000 
[17] C.G.Askins, "Instrumentation for interrogating many-element fiber Bragg grating arrays", SPIE 2444, Smart Sensing, Processing, and Instrumentation, pp. 257-266, 1995 and Private Communication

[18] S.Chen, Y.Hu, L.Zhang, I.Bennion, "Multiplexing of large-scale FBG arrays using a two-dimentional spectrometer" SPIE 3330, Conf. on Sensory Phenomina and Measurement Instrumentation for Smart Structures and Materials, p. 245-252, 1998 and S.Chen, "Large-scale, low cost optical fiber strain sensor arrays", SPIE 4077, pp. 15-26, 2000

[19] M.A.Davis, "A 60 Element Fiber Bragg Grating Sensor System", Proc. Int. Conf. Opt. Fib. Sens. 11 Saporo, Japan, p. 100-103 1996

[20] X.Wan, H.F.Taylor, "Multiplexing of FBG sensors using modelocked wavelength-swept fibre laser" Elec. Lett. 39 (21), pp. ?? 2003

[21] L.Zhang, Y.Liu, J.A.R.Williams, I Bennion, "Enhanced FBG strain sensing multiplexing capacity using combination of intensity and wavelength dual-coding technique", IEEE Phot. Tech. Lett. 11 (12), 1638-1640, 1999

[22] K.P.Koo, A.B.Tveten, S.T.Vohra "DWDM of fiber Bragg grating sensors without sensor spectral dynamic range limitation using CDMA", Optical Fiber Communication Conference, 1999, and the International Conference on Integrated Optics and Optical Fiber Communication. OFC/IOOC '99. Technical Digest ,Volume: 4 , 21-26 Feb. 1999 , pp. 168 - 170, vol.4

[23] C.McGarrity, D.A.Jackson "A network for large numbers of interferometric sensors and fiber Bragg gratings with high resolution and extended range" J.Light.Tech. 16 (1), pp. 54-65, 1998

[24] R.Arai, A.Sumita, S.Makino, T.Maekawa, S.Morimoto, "Large-scale hybrid monitoring system for temperature, strain and vibration using fiber Bragg grating sensors" Advanced Sensor Systems and Applications, SPIE 4920, pp. 62-72, 2002

[25] K.Hotate, M.Enyama, S.Yamashita, Y.Nasu, "High density multiplexing technique of Bragg grating sensors by synthesis of optical coherence function", Advanced Sensor Systems and Applications, SPIE 4920, pp. 285-294, 2002

[26] G.D.Lloyd, L.A.Everall, K.Sugden, I Bennion, "Resonant cavity time-division multiplexed fiber Bragg grating sensor interrogator", IEEE Phot. Tech. Lett. 16(10), 2004, pp. 2323-2325 and www.insensys.com

[27] M.A.Putnam, M.L.Dennis, I.N.Duling III, C.G.Askins, E.J.Friebele, "Broadband square-pulse operation of a passively mode-locked fiber laser for fiber Bragg grating interrogation" Opt. Lett., vol. 23, no.2, 1998, pp. 138-140

[28] G.A.Cranch, C.Kirkendall "Emission Properties of a Passively Mode-Locked Fiber Laser for Time Division Multiplexing of Fiber Bragg Grating Array Applications", Proc. $17^{\text {th }}$ Int. Conf. Optical Fiber Sensors, SPIE 5855, paper 5855-241, Bruges, Belgium, May 2005

[29] C.G.Askins, M.A.Putnam, G.M.Williams, E.J.Friebele, "Stepped-wavelength optical-fiber Bragg grating arrays fabricated in-line on a draw tower" Opt. Lett., vol. 19, no. 2, 1994, pp. 147-149

[30] G.A.Miller, C.G.Askins, P.Skeath, C.C.Wang, E.J.Friebele, "Fabricating fiber Bragg gratings with tailored spectral properties for strain sensor arrays using post-exposure rescan technique" Proc. Int. Conf. Opt. Fib. Sens. 2002, 6-10 May, Portland, Oregon, Post-deadline paper PD1

[31] D.S.Starodubov, V.Grubsky, J.Feinberg, "Efficient Bragg grating fabrication in a fibre through its polymer jacket using near-UV light", Elec. Lett. vol. 33, no. 15, 1997, pp. 1331-1333

[32] V.J.Matsas, T.P.Newson, M.N.Zervas, "Self-starting passively mode-locked fibre ring laser exploiting nonlinear polarization switching" Optics Comm., vol. 92, 1992, pp. 61-66

[33] A.Dandridge, A.B.Tveten, T.G.Giallorenzi, "Homodyne demodulation scheme for fiber optic sensors using phase generated carrier" IEEE $J$ Quant Elec., vol. 18, no. 10, 1982, pp. 1647-1653

[34] C.D.Butter, G.B.Hocker, "Fiber-optic strain gauge" Applied Optics, vol. 17, no. 18, 1978, pp. 2867-2869

[35] A.D.Kersey, T.A.Berkoff, W.W.Morey, "Fiber-optic Bragg grating strain sensor with drift-compensated high-resolution interferometric wavelength-shift detection" Optics Lett., vol. 18, no. 1, 1993, pp. 72-74

[36] M.D.Todd, M.Seaver, F.Bucholtz, "Improved, operationally-passive interferometric demodulation method using 3*3 coupler" Elec. Lett..38 (15) pp. 784-786, 2002

[37] G.A.Cranch, G.M.H.Flockhart, C.K.Kirkendall, "Efficient fiber Bragg grating and fiber Fabry-Pérot sensor multiplexing scheme using a broadband, pulsed mode-locked laser", J.Light. Tech., 23, 11, 2005

[38] G.A.Cranch, G.M.H.Flockhart, C.K.Kirkendall, "Polarization properties of interferometrically interrogated fiber Bragg grating and tandem interferometer strain sensors" accepted for publication in J.Light. Tech. 2006

[39] "Fiber optic smart structures" ed. E. Udd, John Wiley \& Sons, 1995, sec. 2.4.1

[40] G.M. Flockhart, G.A.Cranch, C.K.Kirkendall, "Differential phase tracking applied to Bragg gratings in multicore fiber for high-accuracy curvature measurement" SPIE 6167, San Diego, CA, 26-Feb - 2 Mar, 2006

[41] WB Spillman, RO Claus, "Optical-fiber sensors for the detection of acoustic emission" MRS Bulletin, 27 (5), pp. 396-399, 2002

[42] L.Dong, J.Mistry, "Acoustic emission monitoring of composite cylinders" Comp. Struc. 40 (2), pp. 149-158, 1998

[43] D.C.Betz, G.Thursby, B.Culshaw, W.J.Staszewski, "Acousto-ultrasonic sensing using fiber Bragg gratings" Smart Mat. \& Struc. 12, pp. 122128,2003

[44] I.Read, P.Foote, S.Murray, "Optical fibre acoustic emission sensor for damage detection in carbon fibre composite structure" Meas. Sci. \& Tech. 12, pp. N5-N9, 2002 